\title{
The Impact of Digital Media on Mandala Making in Art Therapy in the Treatment of Anxiety
}

\author{
Elizabeth Donahue $^{1}$ (D) https://orcid.org/0000-0002-6660-8022 \\ Cass Dykeman² ${ }^{(D)}$ https://orcid.org/0000-0001-7708-1409 \\ ${ }^{1}$ Antioch University Seattle \\ ${ }^{2}$ Oregon State University
}

This is the authors' preprint of a manuscript for consideration by a peer reviewed journal. Any comments are invited and can be directed to the first author at edonahue@antioch.edu

\begin{abstract}
In the United States, the usage of electronic media has become ubiquitous. However, the field of art therapy still lags behind in this respect, and it has failed to embrace the use of technology in clinical practice. There exists some evidence that art therapists resist the use of technology in practice because there is little substantiation that digital media is as effective as conventional media in the treatment of mental health issues. Hence, the present study examined if a switch from conventional to digital media impacted the dependent variables (i.e., anxiety, client treatment satisfaction). The experimental design employed was an ABAB reversal design. Results did not indicate this switch had any effect.
\end{abstract}

Keywords: art therapy, digital media, mandala, tablet

\section{Introduction}

Over the last decade, the digital revolution has reshaped all aspects of American culture and economics. In the late 1990s, 37\% of households in the US had a computer and $18 \%$ had access to the internet at home (US Census Bureau, 2012). By 2012, those numbers had increased significantly. The US Census Bureau (2012) reported that by the early 2010 s, $79 \%$ of households had a computer at home and $75 \%$ had access to home internet. As home access to computers and internet has increased, people are finding that their lives are becoming influenced in ever growing ways. The majority of people in the United States are now purchasing movies and music, doing personal banking, and communicating with family and friends via social media right from the comfort of their homes. The Digital Revolution has also influenced the way that we can create art. Whereas conventional media such as canvas, paints, and markers are still widely used, artists are increasingly utilizing the digital media in the form of computers, 
digital cameras and tablets. Also, while this revolution is reshaping society, it has yet to be addressed in art therapy research.

The efficacy of art therapy using traditional media has long been well known (Abbott et al., 2013; Curry \& Kasser, 2005; Slegelis, 1987). One common art therapy intervention is the creation of mandalas. Mandala-making using traditional art media in art therapy sessions has been shown to be an effective intervention in treating a variety of mental health issues. As digital media becomes more pervasive and accepted, it is more frequently used by art therapists in their work with clients because people are increasingly using digital media to express their creativity and meaning-making. However, there has been very little research to date on the efficacy of utilizing digital media. This study is designed to address this research lacuna.

The present study explores the impact of digital media in art therapy regarding adults. A review of the literature revealed four critical topics, which were: (1) Art therapy with conventional media has been well-established as an effective method for the treatment of a variety of mental health issues, (2) In recent years, the field of art therapy has begun to contemplate incorporating digital media into the field, (3) The field of art therapy will benefit from incorporating digital media into practice, and (4) Art therapists may be more likely to incorporate digital media into their clinical practice if it can be demonstrated that it is as effective as the use of conventional media. Finally, the research question will be presented.

Mandala-making with conventional media has been confirmed as an effective method for the treatment of a variety of mental health issues. The key early study on this intervention was conducted by Slegelis (1987) where he found that drawing inside a circle resulted in more positive affect as compared to drawing inside a square.

Henderson, Rosen, and Mascaro (2007) looked at undergraduate students who reported moderate symptoms of Posttraumatic Stress Disorder (PTSD) as measured by the Posttraumatic Stress Disorder Scale (PDS; Foa, 1995). They found that participants who drew a mandala on three consecutive days experienced fewer symptoms of PTSD during a 1-month follow up as compared to participants who drew an object of their choice. Curry and Kasser (2005) examined anxiety levels as measured by the State Anxiety Inventory (Goolkasian, n.d.) in undergraduate students. Participants colored a pre-drawn mandala, a plaid design, or a design of their choice for 20 minutes. Curry and Kasser (2005) found that participants who colored either the mandala or the plaid design experienced decreased levels of anxiety at the end of the intervention.

In recent years, the field of art therapy has begun to envision the integration of digital media into the field. Digital media is defined as separate from conventional art media and includes the use of 
computers and digital tablets such as the iPad or Surface. In 2011, the American Art Therapy Association (AATA) formed the AATA Technology Committee, with an intention to "gather, formulate and disseminate knowledge relevant to the use of digital technology as a therapeutic medium" (AATA, 2011, p. 1). Also, AATA seeks to promote the "safe and ethical use of technology in art therapy" (AATA, 2011, p. 2). Formation of the Technology Committee grew as a consequence of the increased number of conversations about digital art therapy on blogs and at the American Art Therapy Association (AATA) annual conference. In 2009, Orr (2009) conducted a survey of 177 practicing art therapists, and found that while $88 \%$ of art therapists use some form of technology in their practice, only $19 \%$ use technology for art -making with clients. According to Orr (2009) "The participants reported a variety of reasons for not including technology in their practice including limited time for use and training in digital media technology and not being comfortable with the medium" (p. 195). Orr (2009) concluded that there is a significant need for training in digital media technology and that the field of art therapy will benefit from including such training in art therapy programs.

It is obvious that the field of art therapy will benefit from incorporating digital media into practice (Orr, 2009). Failing to do so will result in the practice of art therapy losing relevance in a culture that is steeped in technology (Kapitan, 2009). This is particularly true since too few art therapists are using technology in their practice (Kapitan, 2011). While the use of electronic media has become widespread in the United States, art therapy lags behind. Although many art therapists embrace the use of new technologies in their practice; others are still hesitant and resist the use of digital media in their work (Orr, 2009). Research on the efficacy of digital media in art therapy has been conspicuously limited thus far. This gap in the literature has resulted, according to Kapitan (2011) in "ambivalence and anxiety concerning the power of technology" (p. 50) to flourish in the field of art therapy.

Art therapists may be more likely to incorporate digital media into their clinical practice if it can be demonstrated that it is as effective as the use of conventional media. Reports of the nascent efforts to add digital media to art making with clients are varied. Peterson (2010) surveyed 136 practicing art therapists to learn more about how they incorporate digital media into their practice. Peterson used Rogers (2003) adoption process for media innovation to explore how art therapists adopt new technology. Rogers (2003) posits that there are four elements that influence how people will adopt new technology: the technology itself, the ways in which people communicate about it, time, and the social system within which the technology exists. For a new technology to be sustained in a social 
system, it must be widely adopted. Peterson found that art therapists are likely to adopt new technology when they see it as an improvement upon old technology. For example, art therapists who used phototherapy in their practice quickly moved to the use of Polaroid cameras over conventional film cameras when this new technology became available. While it took days to send out film and have it developed, Polaroid pictures were ready for use within minutes, and art therapists saw this as a big improvement over the old technology (Wolf, 2007).

Although an increasing number of art therapists are integrating digital media into their practice, as compared to the general population, the rate at which they are doing so is curiously slow. There are several reasons that may make art therapists hesitant to include digital media in their repertoire and they include: the high cost of acquisition of software and hardware, the field's regard for traditional media as more beneficial, lack of opportunities for training in the use of digital media, and an understanding that the sensory experience offered by digital media is "cold" and less healing than that offered by conventional media (Carlton, 2014). Finally, there is a dearth of evidence that digital media is as effective as conventional media, which has become an obstacle to art therapists fully accepting and integrating digital media into their practice.

The purpose of this study is to compare the efficacy of conventional art media and digital art media when using a mandala art therapy intervention to treat symptoms of anxiety in adults. There were two research questions which guided this study. The first question was: In reference to GAD symptom level, what is the impact of a switch to digital media for mandala-making in art therapy for clients? The second question was: In reference to treatment satisfaction, what is the impact of a switch to digital media for mandala-making in art therapy for clients?

\section{Method}

\section{Design}

This study used an experimental, non-concurrent multiple baseline single subject research design (Watson \& Workman, 1981). Within single subject research, conventional practices include a minimum of three data points for a baseline phase in order to establish baseline stability (Byiers et al., 2012). For reasons associated with both a field setting and the brief nature of the intervention, one of the three baseline phases was composed of two points. Limitations associated with this two-point baseline phase will be addressed later in the discussion section. This study is employing this design to examine the impact of an intervention over TAU across two behaviors. The 
independent variable in Phase A was the TAU; the independent variable in Phase B was the TAU plus digital media. The two dependent variables were GAD and Client Satisfaction.

\section{Participants}

Data was collected from three adults who self-reported about having experienced symptoms of anxiety. Potential participants who demonstrated a significant level of anxiety as indicated by an average total score of 6 or higher on the GAD-7 were considered for inclusion in this study. Potential participants who were currently taking psychotropic medication, who had participated in mental health counseling in order to treat symptoms of anxiety, or who were participating in ongoing mental health counseling were excluded from this study. Pseudonyms were used to maintain anonymity.

\section{Lulu}

This participant was 53 years old and identified as a white female. Lulu had begun a doctoral program at the beginning of her participation in this study and reported having used touchscreen technology for five years. As a student of art therapy, Lulu was very familiar with techniques and practices of art therapy. Lulu's score of 6 on the GAD-7 (used as a pre-screen) indicating that Lulu experienced mild symptoms of anxiety in the previous two weeks and therefore met the inclusion criteria for this study.

\section{Maria}

This participant was 60 years old and identified as a white female. She was completing her final year of a master's program at the time of this study. Maria reported that she had been using touchscreen technology for four years. As a student of art therapy, Maria was very familiar with techniques and practices of art therapy. Maria scored 14 on the GAD-7 (used as a pre-screen), indicating that she experienced moderate to severe anxiety in the two weeks prior to screening and therefore met the inclusion criteria for this study.

\section{Michaela}

This participant was 51 years old and identified as a white female. She was in her final year of a bachelor's program at the time of this study. Michaela reported that she had been using touchscreen technology for four years. As a student of art therapy, Michaela was very familiar with techniques and practices of art therapy. She scored 15 on the GAD-7 (used as a pre-screener), indicating that Michaela experienced severe symptoms of anxiety in the previous two weeks and therefore met the inclusion criteria for this study. 


\section{Measures}

\section{Demographic Survey}

The survey inquired about the following items: (a) age (b) gender, (c) education level, (d) race/ethnicity, and (e) years of experience using touch screen technology.

\section{Technology Self-Efficacy Scale (TSES)}

The TSES is a 10-item rating scale that measures participants' self-efficacy regarding the use of touch screen technology. The scale uses a 10-point Guttman scale $(1=$ not at all confident to $10=$ totally confident), and was adapted from Compeau and Higgins (1995) Computer-Self Efficacy Scale in order to better capture participants' feelings toward touch screen technology, which has satisfactory discriminant and convergent validity and high composite reliability. The scale is intended to assess a person's confidence in their ability to use unfamiliar technology under a variety of conditions, such as if they have a user's manual at their disposal, if someone demonstrated proper use of the technology, or if they had used similar technology in the past.

\section{Generalized Anxiety Disorder 7-item Scale (GAD-7)}

The GAD-7 is a seven item rating scale that assesses for anxiety. Participants are asked to report how often seven indicators of anxiety have impacted their lives over the past two weeks. A score of $0,1,2$, or 3 is assigned to the response categories of 'not at all', 'several days', 'more than half the days', and 'nearly every day', respectively. The scores of each of the seven items are then added together. A score of 5 or below indicates mild symptoms of anxiety, a score of 6-10 indicates moderate symptoms of anxiety, and a score of 15-18 indicates symptoms of severe anxiety. The GAD has good reliability, as well as criterion, construct, factorial, and procedural validity (Spitzer et al., 2006).

\section{Client Satisfaction Questionnaire 3 (CSQ-3)}

The CSQ-3 is a three item rating scale that measures client satisfaction with the therapeutic process (Larsen, Attkisson, Hargreaves, \& Nguyen, 1979). Participants are asked to indicate their satisfaction in 3 areas on a 4-point, fully anchored Likert scale. The wording of two items was modified to fit an art therapy intervention. Each item queries a participant's opinion on the service received during the session. The overall score is produced by summing all item responses. Total scores range from 3 to 12, with higher scores indicating a higher 
level of participant satisfaction. The CSQ-3 is documented to have excellent reliability and internal consistency (Creamer et al., 2003).

\section{Intervention}

Baseline (Phase A-TAU)

During the Baseline Period (BP), participants met individually with the researcher for one and a half hours each week. Participants were provided with chalk, charcoal, oil pastels, wax crayons, graphite pencils, and colored pencils in a variety of colors and one 8.5" x 11 " sheet of standard drawing paper. In the first session, participants were briefly instructed on how to create a mandala. Participants were then asked to create a mandala, and were told they had approximately half an hour to complete this task. At the end of the hour, the GAD-7 and CSQ-3 were administered.

\section{Intervention (Phase B-Electronic Media)}

During the Electronic Media Period (EMP), participants were provided with an iPad brand digital media tablet with the Art Set Pro app installed and a stylus. Art Set Pro closely replicates drawings and paintings with conventional art materials such as chalk, charcoal, oil pastels, wax crayons, graphite pencils, and colored pencils. During the first session of this period, participants were briefly instructed on the use of the Paper app with the iPad and the stylus. Participants were then asked to create a mandala, and were told they had approximately half an hour to complete this task. At the end of the hour, the GAD-7 and CSQ-3 were administered.

\section{Therapist}

The art therapist was the first author (ED). She is a Licensed Mental Health Counselor in the State of Washington, and a Registered and Board-Certified Art Therapist with the Art Therapy Credentials Board. She received a Master of Arts in Psychology with an Art Therapy Specialization from Antioch University, Seattle, and at the time of the study was a doctoral student in Counselor Education and Supervision at Oregon State University. She has provided art therapy in inpatient psychiatric and private practice settings, working with children and adolescents experiencing severe and persistent mental health issues.

\section{Procedures}

The process of approval for human subject research by Oregon State University and Antioch University Seattle's institutional review board was duly followed and approval was obtained. An advertisement for participants was distributed via email and flyers to art therapy 
students at a private university located in the Western US. Potential participants were prescreened for symptoms of anxiety. Participants endorsed symptoms of anxiety and those who received a score of 10 or higher on the GAD-7 were considered for inclusion in this study.

Participants who were taking psychotropic medication or who were participating in ongoing mental health counseling were excluded from this study.

Three participants met inclusion criteria and were randomly assigned to baseline lengths of two, four, and six weeks. During the baseline phase, clients used conventional media to fill in a pre-drawn circle. At the conclusion of the baseline phase, digital media was introduced in the form of an iPad and a stylus. The iPad was loaded with the Paper 53 app, and a circle approximately 5 " in diameter was pre-drawn on the screen. Participants were instructed to use the stylus to fill in the shape however they wished. Participants drew for 30 minutes. After each session, the GAD-7 was administered to assess for symptoms anxiety and the CSQ-3 was administered to assess each participant's level of satisfaction with the intervention.

The conventional art product was stored in a locked filing cabinet located in the locked office. The digital media remained stored on the iPad, which was password protected and was not synced connected to the Internet. The iPad's Cloud syncing capability was disabled, and it was stored in the same locked filing cabinet in the same locked office as the conventional media.

\section{Apparatus}

\section{Apple iPad}

The Apple iPad Air 2 is a portable handheld device with a touchscreen interface. The iPad measures 0.24 " x 6.60 " x 9.40" and weighs about 445.088 grams. Users may install applications ("apps") which perform a variety of functions including taking and manipulating photographs, playing and creating music, and creating digital art. Users interact with apps using the touchscreen interface. Users may optionally use a stylus to interact with apps.

\section{App}

Participants used the Paper (version 3.6.4) which was released in March 2015. The Paper by FiftyThree app allows users to draw and paint via photo realistic interface with tools that look and feel very much like conventional art media. Users can choose to utilize chalk, charcoal, oil pastels, wax crayons, graphite pencils, and colored pencils. 
Participants were provided with the Pencil by FiftyThree Digital Stylus, model number 53PW06. The stylus allowed participants to draw or paint with more precision than is possible when simply using a finger on the touchscreen. The stylus links via Bluetooth technology to the iPad, which allowed participants to rest their palm on the screen while drawing without inadvertently making accidental marks on their artwork (as is possible with conventional art media). The design of the stylus closely represents that of a carpenter's pencil.

\section{Procedural Fidelity}

A 15-item checklist was created to check for procedural fidelity. Each item on the checklist was observed by the therapist as present or not present. Overall treatment fidelity was calculated by averaging scores for each participant. Treatment fidelity was observed at $100 \%$.

\section{Data Analysis}

Scores on each measure were graphed session by session for the three participants (Spriggs \& Gast, 2010). Visual analysis of the graphs was used to evaluate the data across participants in reference to the stated outcomes (Spriggs \& Gast, 2010). Slope trend analysis for the shift from phases A to B will be completed (Nugent, 2001; Nugent, 2009). Statistical analysis of the data for each dependent variable utilized the Nonoverlap of All Pairs (NAP). According to Parker and Vannest (2009), this method "...summarizes data overlap between each phase A datapoint and each phase B datapoint" (p. 358). NAP for each $\mathrm{AB}$ phase and all $\mathrm{AB}$ phases combined was determined using a web-based NAP Calculator (Vannest, et al.,2016).

\section{Results}

\section{GAD Symptoms}

Figure 1 contains a graphical representation of the results for GAD symptoms. Visual inspection of the Phase A median line and the Phase B median line revealed that two participants improved on GAD symptom as a result of the intervention. The GAD symptoms for one participant remained stable across the phases. In terms of statistical analysis, moderate effect sizes were encountered with the NAP scores for two participants. The NAP score for Participant 1 was .79 and for Participant 2 was .72. The effect size found for Participant 3 was weak $(\mathrm{NAP}=.63)$. In summary, there was no evidence that the switch to digital media had a negative effect on GAD symptoms. 


\section{Client Satisfaction}

Figure 2 presents the results in terms of client satisfaction. Visual inspection of the Phase A median line and the Phase B median line revealed that for all participants, client satisfaction did not change with the switch to digital media. In terms of statistical analysis, the effect size was moderate for Participant 1 (NAP $=.83)$. The NAP scores for Participant 2 and Participant 3 were both in the weak effects range (Participant $2=.44$, Participant $3=.17$ ). In conclusion, the switch to digital media had no effect upon client satisfaction. The average scores for both conventional media $(\bar{x}=10.31)$ and digital media $(\bar{x}=10.47)$ were high.

\section{Discussion}

This study sought to answer two questions. First, what is the impact of a switch to digital media from conventional media when using mandala-making in art therapy when it comes to the reduction of the symptoms of GAD? Second, what is the impact of a switch to digital media from conventional media when using mandala-making in art therapy for clients who are experiencing anxiety in terms of client satisfaction? The results suggest that a switch to digital media from conventional media had no less of a positive effect in the reduction of the symptoms of anxiety, and had little effect in terms of client satisfaction.

Looking first at symptoms of GAD, two participants experienced reduced symptoms of anxiety over the course of the eightweek study. The third participant experienced a very slight reduction in symptoms. The findings suggest that the use of digital media in art therapy may be as effective as the use of conventional media in reducing symptoms. The data shows that a switch from conventional media to digital media did not have negative effect on symptoms of generalized anxiety for any of the participants. Curry and Kasser (2005) showed that creating mandalas with conventional media results in reduced symptoms of anxiety. The results of the current study show that the use of digital media is no less effective than the use of conventional media in art therapy. One alternate explanation for this result is that the study happened to commence at the beginning of an academic quarter and conclude during a break between quarters. All the participants were students at the same university and were enrolled during this academic quarter. In general, most students' anxiety levels are lower during a break in quarters than at the beginning of an academic quarter. It could be that this timing contributed somewhat to reduction in symptoms of anxiety.

Finally, in terms of client satisfaction, the data suggests that

the introduction of digital media had no effect. Participants were very 
satisfied with the intervention irrespective of whether they used conventional media or digital media. These findings show that people are satisfied with their experience when digital media is used as they are with the usage of conventional media in art therapy.

Taken together, the data suggests that digital media is no less effective than conventional media in an art therapy setting. Further, in some cases, digital media may in fact be more effective for some clients that conventional media. As it has been demonstrated here, digital media is at least as effective as conventional media, and clients are very satisfied with both types of media, so art therapists should consider incorporating digital media into their practice. With a dearth of research on the use of digital media in art therapy, many art therapists are resistant to include technology in their practice, believing that conventional media is more therapeutic and more effective that the cold sensory experience of digital media. The results of this study show that the greater efficacy of conventional media may not be the case. Digital media is, in fact, as therapeutic and effective as conventional media.

Limitations to the internal and external validity of this study should be noted. In terms of internal validity, two threats should be noted. These are history and instrumentation (Christ, 2007). In reference to the history threat, all three participants were students at the time of the study. The last sessions occurred during the end of the academic quarter, when students at this institution are generally under less stress than during the rest of the quarter. In reference to instrumentation, a non-standard and unplanned drift to a two-point baseline for the first participant in this study occurred. This drift may have resulted in a slight lack of baseline information for this participant. Also, a stricter adherence to procedural fidelity could have been observed. Though a procedural fidelity checklist was created and used by the researcher, to ensure higher adherence to procedural fidelity it could have been implemented by a colleague or another researcher.

As to external validity, three threats may have influenced the outcomes of the study and thus are potential limitations. Specifically, experimenter effect, novelty, and multiple treatment effect (Bracht \& Glass, 1968). First, there may have been an experimenter effect in that the researcher (who was also the person who delivered the intervention) was a former instructor of each of the three participants. It could be that the previous student-teacher relationship had an effect on the experience of participants during the study. Second, it should be considered that the novelty of the digital media technology may have had an effect. It could be that increased interest in the new media influenced participants' responses on the measures (especially client satisfaction). Finally, a multiple treatment effect should be considered. 
Digital media provides a different haptic experience for the client when compared to conventional media. Conceptually, this different tactile experience could have operated separately from the impact of the switch of media (i.e., conventional to digital). While the more "flat" sensory experience offered by a tablet and stylus may not be an appropriate media choice for every client, some may benefit from the use of more contained and structured media (Hinz, 2009). The data suggests that there was no negative impact on the efficacy of the intervention or on client satisfaction as the result of the haptic experience provided by the iPad and stylus.

Two implications for practice emerged from the results of this study. The first concerns the transfer of knowledge to practice. Rogers' (2003) diffusion of technology theory maintains that for new technology to be sustained in a social system, it must first be widely adopted. Therefore, if digital media is to be fully embraced by the field of art therapy, more art therapists must incorporate the use of digital media in their practice. Peterson (2010), employing Rogers' diffusion technology theory, showed that art therapists are more likely to adopt new technology if they see it as an improvement over old technology. The second imolucation for practice is that digital media has several clear benefits over conventional media. Digital media tools such as an iPad take up less space than the assortment of tools required for conventional art making. The art product of digital media takes up virtually no space, as opposed to conventional two- and threedimensional art products, which effectively solves the issue of storage of client art which faces many practicing art therapists. Digital media tools are more portable than conventional media tools, which can be more convenient for art therapists who travel to multiple settings as part of their practice. Finally, the use of digital media requires essentially zero clean-up, while the use of conventional media tools can result in at least some cleanup time after each art therapy session. As more art therapists begin to realize these benefits of digital media over conventional media, the rate of adoption of this new technology may increase.

The results of this study set a foundation for future research on the efficacy of digital media in art therapy. With this new evidence that digital media does not hamper the therapeutic nature of art making, and as client satisfaction with the medium is high, perhaps art therapists will be more likely to implement the use of this technology in their practice.

At the time of this writing, there was no published quantitative data on the use of digital media in art therapy to treat symptoms of anxiety or on the effect of client satisfaction with art therapy when using digital media as compared to conventional media. Further research must be conducted on a variety of populations (older 
adults, children, adolescents, or those with physical limitations which make the use of conventional media challenging would all be good places to start). Future studies on the use of digital media in art therapy in the treatment of other diagnoses (depression, gender identity disorder, or PTSD for example) is also needed.

In conclusion, the results of this study show that the use of digital media is no less effective than the use of conventional media when using mandala-making in the treatment of symptoms of anxiety in an art therapy setting. In addition, client satisfaction remains high when digital media is introduced over conventional media. These findings may encourage art therapists to include digital media in their future practice.

\section{References}

Abbott, K. A., Shanahan, M. J., \& Neufeld, R. W. (2013). Artistic tasks outperform nonartistic tasks for stress reduction. Art Therapy: Journal of the American Art Therapy Association, 30, 71-78. http://dx.doi.org/10.1080/07421656.2013.787214

Bracht, G. H., \& Glass, G. V. (1968). The external validity of experiments. American Educational Research Journal, 5, 437474. https://doi.org/10.3102/00028312005004437

Carlton, N. R. (2014, February). Digital culture and art therapy. The Arts in Psychotherapy, 41, 41-45. http://dx.doi.org/10.1016/j.aip.2013.11.006

Christ, T. J. (2007). Experimental control and threats to internal validity of concurrent and nonconcurrent multiple baseline designs. Psychology in the Schools, 44, 451-

459. doi:10.1002/pits.20237

Creamer, M., Bell, R., \& Failla, S. (2003, December). Psychometric properties of the Impact of Event Scale-Revised. Behaviour Research and Therapy, 41(12), 1489-1496.

http://dx.doi.org/2003.07.010

Curry, N. A., \& Kasser, T. (2005). Can coloring mandalas reduce anxiety? Art Therapy: Journal of the American Art Therapy Association, 22, 81-85. http://dx.doi.org/10.1080/07421656.2005.10129441

Foa, E. B. (1995). PDS. Posttraumatic Stress Diagnostic Scale: Manual. Minneapolis, MN: National Computer Systems, Inc.

Goolkasian, P. (n.d.). Spielberger's State Anxiety Inventory [Measurement instrument]. http://www.mindgarden.com/products/staisad.htm

Henderson, P., Rosen, D., \& Mascaro, N. (2007). Empirical study on the healing nature of mandalas. Psychology of Aesthetics, Creativity, and the Arts, 1, 148-154. 
Hinz, L. D. (2009). Expressive therapies continuum: A framework for using art in therapy. Routledge.

Kapitan, L. (2007). Will art therapy cross the digital culture divide? Art Therapy: Journal of the American Art Therapy Association, 24(2), 50-51. http://dx.doi.org/10.1080/07421656.2007.10129591

Kilpatrick, D. G., Resnick, H. S., \& Friedman, M. J. (2013). Severity of Posttraumatic Stress Symptoms-Adult *National Stressful Events Survey PTSD Short Scale (NSESSS) [Measurement instrument]. Unpublished instrument. www.psychiatry.org

Larsen, D. L., Attkisson, C. C., Hargreaves, W. A., \& Nguyen, T. D. (1979). Assessment of client/patient satisfaction: development of a general scale. Evaluation and Program Planning, 2, 197207. http://dx.doi.org/10.1016/0149-7189(79)90094-6

Munder, T., Wilmers, F., Leonhart, R., Linster, H. W., \& Barth, J. (2010). Working Alliance Inventory-Short Revised (WAI-SR): psychometric properties in outpatients and inpatients. Clinical Psychology \& Psychotherapy, 17(3), 231-239. http://dx.doi.org/10.1002/cpp.658

Orr, P. P. (2009). Technology training for future art therapists: Is therea need? Art Therapy: Journal of the American Art Therapy Association, 23(4), 191-196. http://dx.doi.org/10.1080/07421656.2006.10129329

Parker, R. I., Vannest, K. J., Davis, J. L., \& Sauber, S. B. (2011). Combining nonoverlap and trend for single-case research: TauU. Behavior Therapy, 42(2), 284-299. http://dx.doi.org/10.1016/j.beth.2010.08.006

Peterson, B. C. (2010). The media adoption stage model of technology for art therapy. Art Therapy: Journal of the American Art Therapy Association, 27(1), 26-31. http://dx.doi.org/10.1080/07421656.2010.10129565

Rogers, E. M. (2003). Diffusion of innovations. Simon and Schuster. Scruggs, T. E., \& Mastropieri, M. A. (1998). Summarizing single subject research issues and applications. Behavior Modification, 22(3), 221-242.

Slegelis, M. H. (1987). A study of Jung's mandala and its relationship to art psychotherapy. The Arts in Psychotherapy, 14(4), 301311. http://dx.doi.org/10.1016/0197-4556(87)90018-9

Spitzer, R. L., Kroenke, K., Wiliams, J. B., \& Lowe, B. (2006, May 22). A brief measure for assessing generalized anxiety disorder: The GAD-7. Archives of Internal Medicine, 166(10). Retrieved from www.patient.co.uk

Spriggs, A. D., \& Gast, D. L. (2010). Visual representation of data. Single Subject Research Methodology in Behavioral Sciences, 166-198. 
United States Census Bureau. (2012). Households with a computer and internet Use: 1984 to 2012 [Table 4]. http://www.census.gov/

Vannest, K. J., Parker, R. I., \& Gonen, O. (2011). Single Case Research: web based calculators for SCR analysis. College Station, TX: Texas A\&M University. http://dx.doi.org/dx.doi.org/10.1080/17454830600674050

Watson, P. J., \& Workman, E. A. (1981). The non-concurrent multiple baseline across-individuals design: An extension of the traditional multiple baseline design. Journal of Behavior Therapy and Experimental Psychiatry, 12(3), 257-259. http://dx.doi.org/10.1016/0005-7916(81)90055-0

Wolf, R. I. (2007). Advances in phototherapy training. The Arts in Psychotherapy, 34(2), 124-133.

http://dx.doi.org/10.1016/j.aip.2006.11.004 
Figure 1

Symptoms of Generalized Anxiety

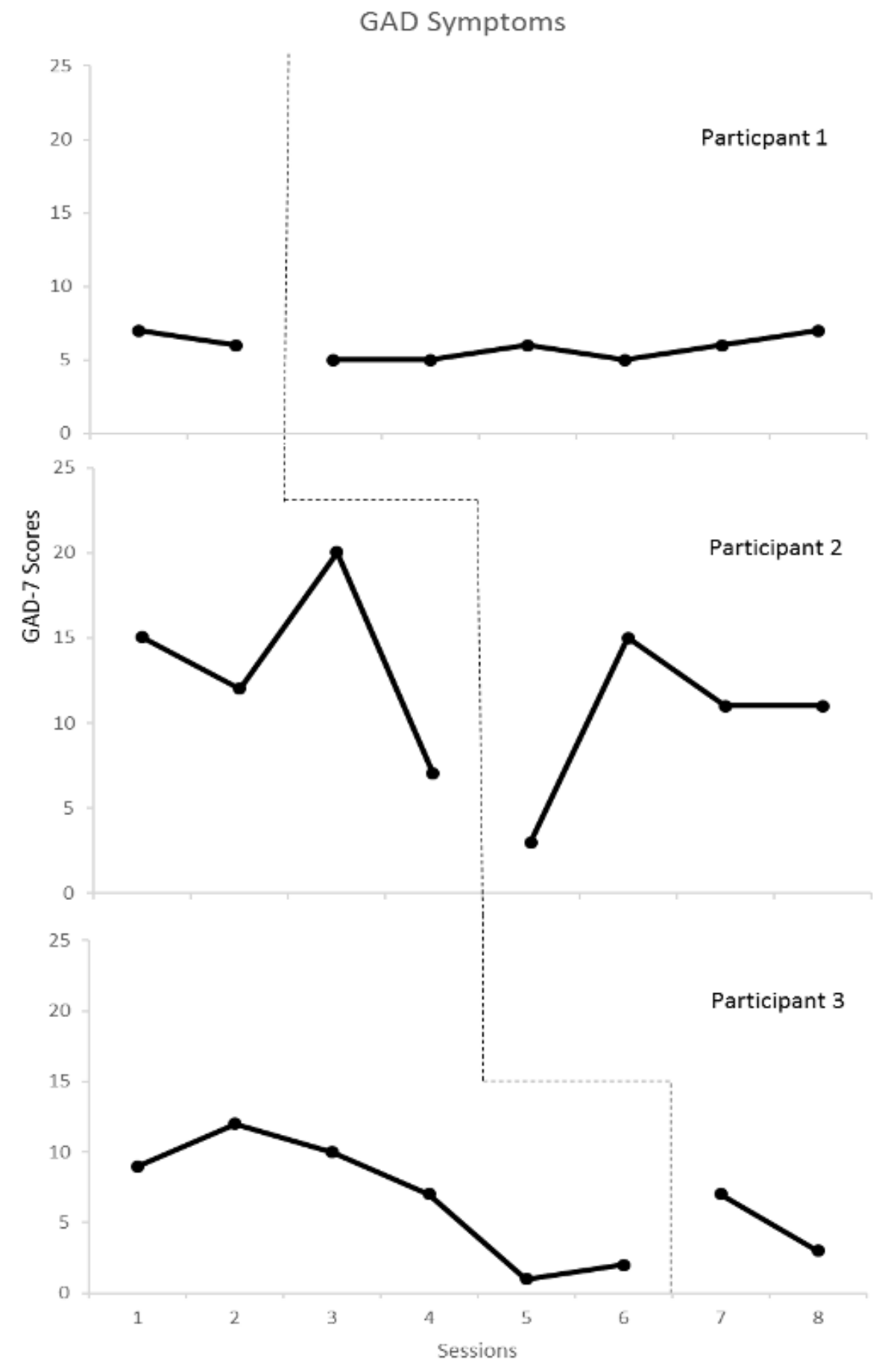


Figure 2

Client Satisfaction

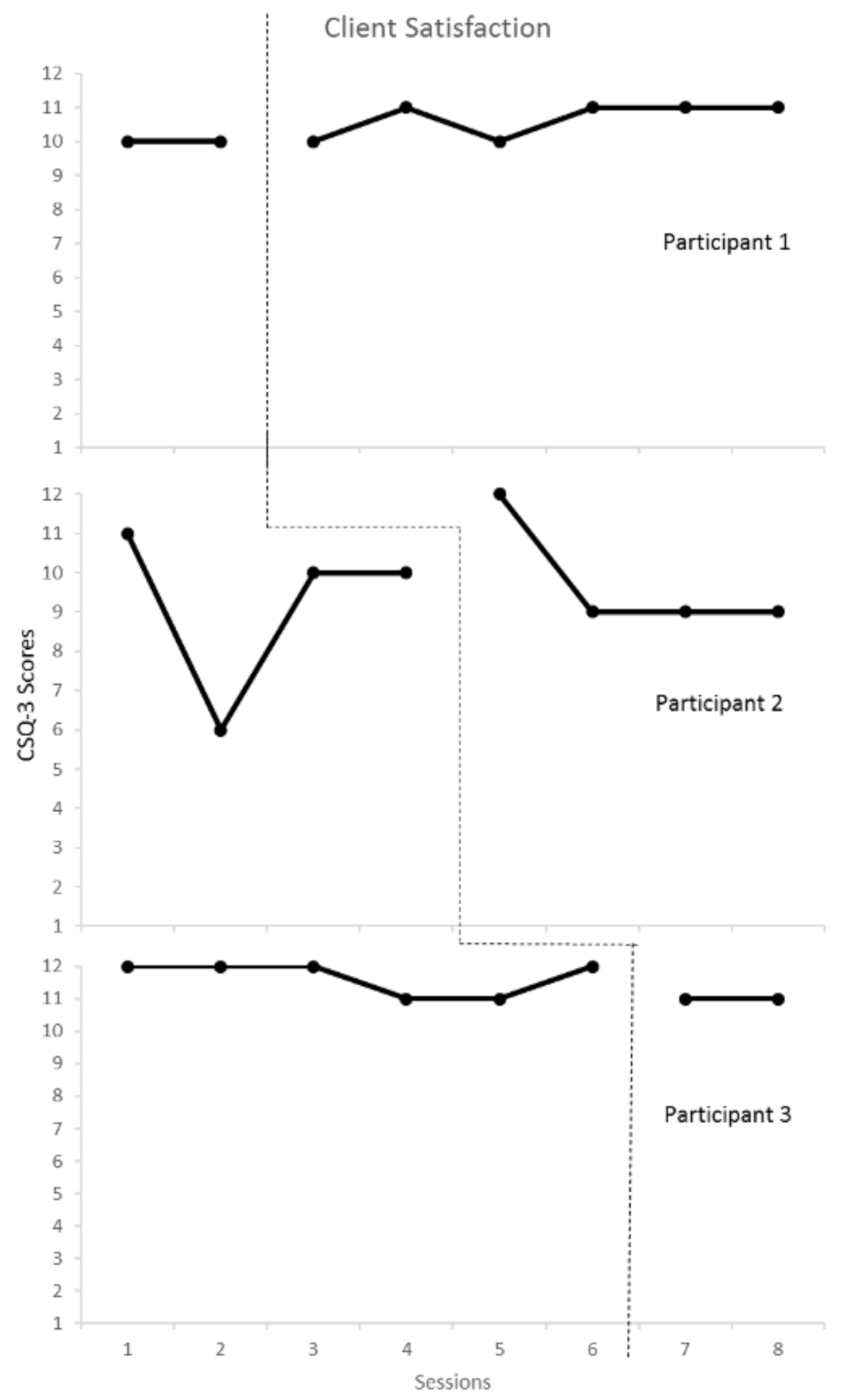

